\author{
SOBRECARGA EM CUIDADORES INFORMAIS DE DEPENDENTES DE \\ SUBSTÂNCIAS: ADAPTAÇÃO DO CAREGIVER REACTION ASSESSMENT \\ (CRA) \\ M. Graça Pereira (gracep@ psi.uminho.pt) \& António José Soares \\ Escola de Psicologia, Universidade do Minho, Braga, Portugal.
}

\begin{abstract}
RESUMO: Os problemas causados pelo abuso e dependência de substâncias assumem-se como um dos mais difíceis de resolver nas sociedades ocidentais. Os cuidadores informais dos Dependentes de Substâncias prestam cuidados que, por vezes, interferem com aspectos da vida pessoal, familiar, laboral e social dos cuidadores, predispondo-os a conflitos. Frequentemente, estas pessoas entram em situações de crise manifestando sintomas como: tensão, constrangimento, fadiga, stress, frustração, redução de convívio, depressão e alteração da auto-estima, entre outros. Esta sobrecarga (burden) ou tensão pode acarretar problemas físicos, psicológicos, emocionais, sociais e financeiros, que acabam por afectar o bem-estar do doente e cuidador. O Caregiver Reaction Assessment (Given, et al., 1992) é um instrumento que avalia aspectos específicos da situação de cuidar, incluindo as dimensões positivas e negativas das reacções dos cuidadores no cuidar de um paciente com uma doença, podendo ser utilizado como medida de sobrecarga. Este artigo apresenta a adaptação do CRA numa amostra de cuidadores de Dependentes de Substâncias. Os resultados mostram uma elevada fidelidade, sendo que três factores explicam $58,76 \%$ da variabilidade total. A subescala Auto-Estima apresenta um $\alpha=0,95$, a subescala Impacto na Vida Familiar do Cuidador um $\alpha=0,88$ e a subescala Impacto no Quotidiano do Cuidador um $\alpha=0,70$. Os 24 itens do instrumento apresentam um $\alpha=0,95$, confirmando as boas qualidades psicométricas e mantendo todos itens originais. Os resultados obtidos com o CRA sugerem uma boa validade de construto, estando negativamente correlacionado com o Suporte Social (IESSS). Com base nos resultados obtidos, pode-se concluir que a escala apresenta boas qualidades psicométricas para ser utilizada numa população de cuidadores de Dependentes de Substâncias., sendo útil como medida de sobrecarga.

Palavras-chave: Sobrecarga; Cuidador Informal; Dependência de Substâncias.
\end{abstract}

\title{
SOBRECARGA EM CUIDADORES INFORMAIS DE DEPENDENTES DE SUBSTÂNCIAS: ADAPTAÇÃO DO CAREGIVER REACTION ASSESSMENT (CRA)
}

ABSTRACT: Problems caused by drug and alcohol addiction poses are the most difficult to handle in western societies. Caregiving interferes with caregiver's own personal, family, job and social life, making them more susceptible to interpersonal conflicts. As a result, caregivers often experience feelings of tension, fatigue, uneasiness, stress, frustration, depression, reduced social interactions, changes in self-esteem, among other symptoms. These feelings of burden and stress may convey physical, psychological, emotional, social and financial problems, ultimately affecting the caregiver and patient's well-being. The Caregiver Reaction Assessment (Given, et al., 1992) is an instrument that assesses specific aspects of caregiving, including positive and negative caregiver reactions, posing as a burden measure. This article presents the adaptation of CRA in a sample of addict's informal caregivers. The results show that the Portuguese adaptation has good validity, with three factors explaining $58,76 \%$ of the observed variance. The Self-Esteem, Impact on Caregiver's Family Life, Impact on Caregiver's Health subscales have internal consistency scores of 95, 88 and, 70 , respectively. The total 24 items of CRA have an internal consistency score of 95 , confirming the 
good psychometric quality of the instrument. The CRA presents good construct validity, being negatively correlated with Social Support (IESSS). Based on these results, $t$ the scale presents good psychometric qualities meeting the terms for the use on drug and alcohol addict's informal caregivers as a burden measure.

Key-Words: Addiction, Burden, Informal Caregiver.

Recebido em 15 de Dezembro de 2009/ Aceite em 30 de Outubro de 2011

A primeira referência sobre o sofrimento dos familiares aparece em 1946 com Treudley que introduziu o conceito de burden na literatura psiquiátrica inglesa. O carácter pejorativo associado à ideia de fardo e a ampla utilização de peso com medida física levou-nos a considerar que a melhor tradução para o termo burden é sobrecarga (Bandeira \& Barroso, 2005).

O conceito de sobrecarga pode ser definido como o impacto negativo num (ou mais) determinado membro da família (cuidador), em resultado da prestação das tarefas inerentes aos cuidados necessários para que um outro membro da família (doente) possa lidar com a sua patologia. Esta sobrecarga também pode advir das alterações psicológicas/comportamentais, directa ou indirectamente, relacionadas com a doença do membro da família. O impacto das diversas condições médicas atinge um amplo espectro de dimensões da vida familiar e pode ser considerado sobrecarga porque requer que os familiares do paciente passem a colocar as suas próprias necessidades e desejos em segundo plano (Kalra, Kamath, Trivedi, \& Janca, 2008; Maurin \& Boyd, 1990) e adoptem um papel suplementar aos já desempenhados por eles afectando a sua qualidade de vida (Bandeira \& Barroso, 2005; Khan, Pallant, \& Brand, 2007; Kochar, Fredman, Stone, \& Cauley, 2007; Martens \& Addington, 2001; Martins, Ribeiro, \& Garrett, 2003).

O termo sobrecarga é composto por uma dimensão objectiva e outra subjectiva (Lauber, Eichenberger, Luginbühl, Keller, \& Rössler, 2003; Martens \& Addington, 2001; Maurin \& Boyd, 1990). A sobrecarga objectiva diz respeito às consequências negativas concretas e observáveis resultantes da presença do doente na família, tais como perdas financeiras, perturbações na rotina, na vida social e na vida profissional dos familiares, tarefas quotidianas adicionais que precisam executar para cuidar de todos os aspectos da vida do paciente (higiene, transporte, controlo da medicação, alimentação, lazer, finanças, compras, idas aos tratamentos médico, etc.), perturbações nas relações entre os membros da família, ocorrência de comportamentos problemáticos com os quais os familiares precisam lidar (por exemplo, comportamentos embaraçosos, agressões físicas e verbais, condutas sexuais inadequadas, comportamentos que perturbam a rotina da casa, ameaças ou tentativas de suicídio, abuso de álcool e drogas, etc.) (Bandeira \& Barroso, 2005; Martens \& Addington, 2001; Martins, et al., 2003; Maurin \& Boyd, 1990; Reine, et al., 2004; Tessler \& Gamache, 2000).

O aspecto subjectivo da sobrecarga refere-se à percepção ou avaliação pessoal do cuidador sobre a situação, envolvendo ainda a sua reaç̧ão emocional, atribuída por ele à 
presença do doente na família. Portanto, este aspecto refere-se ao grau em que os familiares percebem a presença, os comportamentos ou a dependência dos pacientes como uma fonte de pensamentos e sentimentos negativos, preocupações e/ou tensão psicológica (Martins, et al., 2003; Maurin \& Boyd, 1990; St. Onge \& Lavoie, 1997). Envolve sentimentos de desamparo, tristeza, culpa, assim como de perda semelhante ao luto. Para além disso, a natureza e o estigma de algumas doenças (mentais, dependência de substâncias) podem provocar sentimentos de constrangimento e exacerbar o isolamento (Bandeira \& Barroso, 2005; Tessler \& Gamache, 2000). Parece que mesmo a comunidade médica não está preparada para lidar com este aspecto (Riebschleger, et al., 2008).

As mudanças que ocorrem no relacionamento do paciente com os seus familiares em consequência da doença, em particular a falta de reciprocidade/comunicação, prejudicam a relação e tornam mais difícil a manutenção de sentimentos positivos no dia-a-dia, o que contribui para o aumento da sobrecarga subjectiva. No caso de um cônjuge que cuida do companheiro doente, essa falta de reciprocidade pode provocar uma deterioração que pode resultar frequentemente em separação/divórcio (Dekel \& Solomon, 2007; Wolff, et al., 2006). Para além disso, um outro factor que agrava a sobrecarga sentida pelos familiares é o facto das tarefas e os cuidados com o paciente ocorrem muitas vezes num momento inesperado, desfasado com o ciclo da vida familiar (Carter \& McGoldrick, 1980), pois normalmente espera-se que o indivíduo, atingindo a idade adulta, seja independente e não necessite de mais de cuidados intensivos. A doença de um dos membros da família quebra essa expectativa e altera as fases do ciclo familiar, criando uma função adicional que não era esperada para aquele momento do ciclo familiar podendo ser considerado um stressor para os familiares (Bandeira \& Barroso, 2005; Tessler \& Gamache, 2000).

Um conjunto significativo de estudos tem demonstrado consistentemente que a presença de um doente mental/físico na família resulta numa considerável sobrecarga para os seus membros. Esta sobrecarga pode estar inclusivamente associada a perturbações psicológicas no cuidador primário (Croog, Burleson, Sudilovsky, \& Baume, 2006; Garand, Eazor, Dew, DeKosky, \& Reynolds, 2005; Jungbauer \& Angermeyer, 2002; Lauber, et al., 2003; Loukissa, 1995; Magliano, et al., 2000; Marvardi, et al., 2005; Rose, 1996).

No caso das doenças crónicas, o cuidador é submetido ao efeito prolongado de eventos stressores envolvidos na experiência quotidiana de cuidar do paciente, o que pode afectar a própria saúde mental (Awad \& Voruganti, 2008; Beckham, Lytle, \& Feldman, 1996; Martens \& Addington, 2001; Rammohan, Rao, \& Subbakrishna, 2002; Schulze \& Rössler, 2005).

Alguns autores destacam as medidas de sobrecarga subjectiva enquanto outros focam-se na sobrecarga objectiva. Maurin e Boyd (1990) referem que a sobrecarga afecta a saúde mental dos familiares, com destaque para as medidas de sobrecarga subjectiva. Segundo a pesquisa de McGilloway et al. (1997), numa amostra com 38 famílias, a maioria dos familiares que cuidavam dos pacientes sofria de transtornos psicológicos, em particular as mulheres. Na 
pesquisa de St. Onge e Lavoie (1997), com uma amostra de 99 cuidadores, a presença de perturbações psicológicas nas mães de pacientes psicóticos era duas vezes maior do que na população geral. De acordo com os autores, esses transtornos resultaram da sobrecarga sentida pela família por ter que cuidar dos seus filhos doentes mentais. Demi et al. (1997), ao estudar 156 cuidadores de mulheres infectadas com HIV, verificaram que o único factor preditor de sobrecarga e depressão era o estigma associado à condição das pacientes. Assim os autores concluiram que, para diminuir o burden, deverão ser empreendidos esforços para reduzir o estigma associado à patologia. Green (2007) estudou a sobrecarga em mães de crianças com deficiência e também verificou que a experiência de burden estava relacionada fortemente com o estigma associado à patologia. Martire et al. (2008) estudaram os cuidadores de pacientes com Depressão. Numa amostra com 130 participantes, verificaram que a sobrecarga previa uma maior severidade dos sintomas depressivos no paciente na sexta semana de tratamento, mesmo controlando as características dos pacientes anteriores ao tratamento, os sintomas depressivos do cuidador e a satisfação com a relação entre cuidadores e pacientes.

Num estudo interessante, O'Rourke e Tuokko (2004) demonstraram numa amostra de 137 cuidadores, que a sobrecarga antecede a depressão e não inversamente. Grov et al. (2006) acompanharam cuidadores de doentes oncológicos na fase paliativa e verificaram que a depressão era o factor principal associado à sobrecarga mas também um factor importante na mediação da relação indirecta entre a saúde física e a ansiedade do cuidador. Pickett (2007) encontrou resultados semelhantes numa amostra de cuidadores de doentes com doença de Huntington.

Meiland et al. (2001) estudaram a percepção de sobrecarga dos cuidadores de pacientes com Demência no momento em que são admitidos na lista de espera de uma instituição para internamento dos seus familiares. Verificaram que metades dos 93 participantes exibiam sinais de sobrecarga. Os factores associados a mais experiências negativas relatadas pelos cuidadores eram: uma Demência menos severa, idade menor do paciente e providenciar mais horas de cuidado informal. A auto-estima derivada dos cuidados era superior em cuidadores com menores rendimentos e naqueles que percepcionavam a qualidade das relações com o paciente como melhores. Black e Almeida (2004) realizaram uma meta análise dos estudos com cuidadores de pacientes com Demência e verificaram que os sintomas comportamentais e psicológicos da Demência (BPSD) eram um preditor de sobrecarga, de distress psicológico e depressão nestes cuidadores. Gómez-Ramos e González-Valverde (2004) encontraram resultados semelhantes.

Marsh et al. (2002) avaliaram 52 cuidadores de pacientes com TCE em dois momentos (6 meses e 1 ano após a lesão) em termos de sobrecarga objectiva e subjectiva. Verificaram que 6 meses após a lesão 1/3 dos cuidadores apresentavam níveis clinicamente significativos de depressão e ansiedade e baixo ajustamento social. Um ano após a lesão a prevalência de depressão e ansiedade manteve-se constante enquanto apenas 1/4 dos cuidadores apresentavam 
baixo ajustamento social. Deste modo, enquanto as medidas de sobrecarga objectiva se mantêm estáveis, alguns aspectos da sobrecarga subjectiva vão diminuindo. Apesar disso, os problemas comportamentais e cognitivos do paciente contribuem numa escala maior para o distress sentido pelos cuidadores e o isolamento social do paciente surge como o factor mais estável e consistente na experiência de sobrecarga subjectiva durante o primeiro ano após a lesão. Jacobi et al. (2003) concluíram igualmente que os parâmetros objectivos e subjectivos de saúde do cuidador e do paciente são relevantes na predição de burden subjectivo. Stancin et al. (2008) acompanharam cuidadores de crianças com TCE e encontraram resultados diferentes. Assim, para além de verificarem maiores índices de sobrecarga e distress nos cuidadores também encontraram níveis clínicos de depressão e distress nos irmãos dos pacientes. Para além disso, estes indicadores estavam associados à gravidade do traumatismo.

Belasco e Sesso (2002) estudaram os cuidadores de pacientes que estavam a realizar hemodiálise há pelo menos 4 meses. Numa amostra com 100 cuidadores, conseguiram identificar os preditores objectivos de sobrecarga. A saúde mental do cuidador, a vitalidade do paciente, ser esposa do paciente e a dor do cuidador, foram identificados como factores independentes e significativos. Andrieu et al. (2003) estudaram os factores objectivos relacionados com a sobrecarga uma amostra de 513 cuidadores de pacientes com Alzheimer. Verificaram que o burden estava relacionado directamente com os comportamentos disruptivos e o impedimento cognitivo do paciente. No entanto, nem a duração da doença nem a disfunção nas actividades do dia-a-dia estavam relacionadas com a sobrecarga. Bruce et al. (2008) encontraram resultados contraditórios em cuidadores de pacientes com impedimentos cognitivos moderados. Neste caso, a sobrecarga estava associada a um maior curso de tempo de problemas cognitivos e a maior depressão nos pacientes apesar dos problemas comportamentais, de humor e de memória também contribuírem para o burden. Bédard et al. (2005) analisaram os factores relacionados coma sobrecarga em cuidadoras de doentes de Alzheimer e verificaram que 46\% da variância era explicada pelos problemas de comportamento e pela dependência instrumental das actividades do dia-a-dia, sendo que as cuidadoras que cuidavam de homens apresentavam 5,61 pontos na escala de burden a mais que os outros cuidadores. Os problemas tidos como mais problemáticos pelas cuidadoras estavam relacionados com problemas específicos (por ex: lidar com o comportamento agressivo). Ohaeri (2003) realizou uma revisão dos estudos que procuraram prever a sobrecarga em cuidadores de pacientes com desordens do foro mental. Verificou que os preditores mais importantes eram o comportamento problemático, a deficiência/impedimento físico e psicológico e a gravidade dos sintomas. Pinquart e Sorensen (2003) numa meta análise de 228 estudos verificaram que os problemas comportamentais dos pacientes apresentavam associações mais fortes com o burden dos cuidadores que os restantes stressores.

Num estudo desenvolvido por Edwards e Scheetz (2002) com cuidadores de pacientes de Parkinson, os autores verificaram que as actividades do dia-a-dia, a percepção do suporte 
social, o bem-estar e a satisfação marital eram preditores de sobrecarga. Sendo que as actividades do dia-a-dia e a percepção do suporte social explicavam 44,3\% da variância. Shrag et al. (2006) chegaram a conclusões semelhantes. Chappell et al. (2002) chegaram a uma conclusão ligeiramente diferente. Num estudo com cuidadores de pacientes com Demência e outras patologias, foram distinguidos os conceitos de burden e bem-estar. Assim, foi elaborado um modelo no qual o bem-estar foi directamente afectado pela percepção de suporte social, pela sobrecarga, auto-estima e horas dispendidas nos cuidados informais, enquanto o burden foi afectado pelos problemas comportamentais, pela frequência dos períodos de descanso, pela auto-estima e pelas horas dispendidas nos cuidados. Deste modo, os autores concluem que o facto de a percepção do suporte social estar fortemente relacionada com o bem-estar mas não relacionada com o burden, é um indicador da distinção dos dois conceitos. McClendon e Smyth (2002) chegaram a conclusões semelhantes.

Goldstein et al. (2004) estudaram cuidadores de doentes oncológicos terminais. Numa amostra com 206 cuidadores verificaram que aqueles que apresentavam maior sobrecarga também possuíam redes sociais mais limitadas, mais restrições das actividades do dia-a-dia e eram mais novos. Os autores concluem que as variações existentes entre os valores de sobrecarga se devem às experiências subjectivas e ao suporte social e não tanto à quantidade de assistência providenciada. Magliano et al. (2004) estudaram cuidadores de diversas patologias físicas crónicas. Numa amostra de 646 cuidadores, desenvolvido em 30 regiões diferentes da Itália, verificaram que os constrangimentos relatados mais frequentemente estavam relacionados com o sentimento de perda e alterações nas actividades sociais e recreativas. A sobrecarga era mais evidente em cuidadores com menor suporte social e de pacientes com maiores dificuldades físicas e com doenças neurológicas. Post et al. (2005), numa amostra com 265 díades cuidadordoente com lesão da coluna vertebral, verificaram que os preditores de sobrecarga eram a quantidade de apoio às actividades do dia-a-dia, os problemas psicológicos do paciente, a sua idade, género, o grau de impedimento físico e o tempo decorrido da lesão.

Andrén e Elmståhl (2008) verificaram existir diferenças nos cuidadores de pacientes com Demência, em termos dos factores associados à sobrecarga. Assim, os cuidadores que experienciavam uma sobrecarga moderada, também exibiam maior isolamento, sentimentos de desapontamento e envolvimento emocional com a percepção da saúde, enquanto os cuidadores com baixa sobrecarga exibiam níveis significativamente melhores de saúde percepcionada. Aliás a percepção de saúde tem sido relacionada negativamente com a sobrecarga por diversos autores noutras populações (por ex: Andrén \& Elmståhl, 2007; La Parra, 2001; Yeh, Johnson, \& Wang, 2002).

A Dependência de Substâncias, assim, como muitas doenças crónicas ou com cariz crónico tem sempre associado uma deterioração das capacidades físicas, cognitivas, emocionais e sociais do indivíduo. Esta vivência da situação de doença é muitas vezes sentida como uma situação de crise, um acontecimento stressor, que tem consequências no doente e na sua família. 
Contudo, cuidar de um familiar debilitado e/ou dependente representa igualmente retribuir sentimentalmente a quem de alguma forma também cuidou de nós e a quem se dedica um afecto profundo ou o cumprimento de uma "obrigação moral". Daí que esta situação também integre aspectos positivos gerados pela solidariedade, proximidade e intimidade (Paúl, 1997). O suporte social, a rede social formal e informal representada por instituições, serviços oficiais públicos ou privados, rede de amigos e voluntários, desempenham um papel importante, pois constituem factores facilitadores que ajudam o cuidador podendo contribuir para a diminuição da sobrecarga (Martins, et al., 2003).

Dado que em Portugal, não existem estudos sobre sobrecarga em cuidadores de Dependentes de substâncias, o objectivo do presente trabalho foi a adaptação do Caregiver Reaction Assessment (CRA), instrumento que avalia a sobrecarga, numa amostra de cuidadores de Dependentes de Substâncias.

\section{MÉTODO}

\section{Amostra}

Este estudo foi realizado com 120 indivíduos. Trata-se de uma amostra de conveniência, composta por 91 mulheres (75,8\%), apresentando uma média etária de 51,51 anos (D.P. 11,99). A maioria é casada (76,7\%), seguidos dos divorciados (12,5\%), viúvos $(5,8 \%)$ e em menor número dos solteiros (5\%). No que diz respeito às habilitações literárias, a maioria possui o ensino secundário (33,3\%), seguido do ensino básico (22,5\%), ensino preparatório $(20,8 \%)$, ensino superior $(17,5 \%)$ e ensino médio/curso profissional $(5,8 \%)$. No que diz respeito ao estado de saúde, 33,3\% dos participantes apresenta doenças da síndrome metabólica (colesterol elevado, diabetes, hipertensão, etc.), sendo que $56,7 \%$ de todos os participantes toma medicação psicofarmacológica. Em relação ao paciente cuidado, é predominantemente o filho(a) $(62,5 \%)$ ou cônjuge (25,8\%), do sexo masculino (80,8\%), com uma média de idade de 34,08 (D.P. 8,09), solteiro $(55,0 \%)$ ou casado/união de facto $(39,2 \%)$, com habilitações literárias ao nível do ensino secundário $(37,5 \%)$ ou preparatório $(24,2 \%)$, está empregado $(61,7 \%)$ mas dependente financeiramente $(58,3 \%)$ e mora com a cuidadora (69,2\%). A Dependência de Substâncias já foi diagnosticada há mais de 5 anos em $79 \%$ dos casos, tendo os pacientes realizado em média 1,81 (D.P. 1,53) internamentos em comunidades terapêuticas, 1,52 (D.P. 1,99) desintoxicações em clínicas e 2,84 (D.P. 6,45) desintoxicações em casa. No que diz respeito à abstinência do uso de substâncias, 37,5\% dos pacientes não está em abstinência, 33,3\% está há menos de 5 anos e $29,2 \%$ está há mais de 5 anos. 10,8\% já estiveram (ou estão) detidos em resultado do abuso de substâncias e 55,8\% tem problemas de saúde graves (HIV/SIDA, Hepatite C, Tuberculose) devido ao abuso de substâncias.

\section{Material}


Para avaliar a sobrecarga do cuidador, utilizou-se a versão de investigação do Caregiver Reaction Assessment (Pereira \& Sousa, 2001). Trata-se de um instrumento que avalia aspectos específicos da situação de cuidar, incluindo as dimensões positivas e negativas das reacções dos cuidadores (Given et al., 1992). Foi desenvolvido por etapas, tendo sido iniciado com 101 itens baseados numa extensa revisão bibliográfica e entrevista a cuidadores. Depois, foi administrado a uma amostra de 99 cuidadores, do qual a análise psicométrica revelou 40 itens. Por fim, após a análise factorial de uma amostra de 377 cuidadores de pacientes com Demência, o instrumento foi reduzido a 24 itens. Assim, a versão original final é constituída por 24 itens que avaliam as reacções do cuidador no cuidar de um paciente com uma doença física. Os itens estão agrupados em 5 dimensões: Auto Estima do cuidador (avalia o valor atribuído ao cuidar como resultado da experiência de ser retribuído ou causar ressentimento - 7 itens - Dimensão Positiva), Falta de Suporte Familiar (avalia a percepção do cuidador de ser o único com responsabilidade nos cuidados do doente ou a percepção de que a família trabalha em conjunto - 5 itens - Dimensão negativa), Impacto nas Finanças (avalia quão adequados ou difíceis são os seus recursos financeiros para cuidar do familiar -3 itens - Dimensão negativa), Impacto nos Horários (avalia qual a extensão de interrupções necessárias para cuidar do familiar e até que ponto isso interfere nas actividades diárias - 5 itens - Dimensão negativa), Impacto na Saúde do Cuidador (avalia a capacidade de cuidar e interferência na saúde do cuidador - 4 itens - Dimensão negativa). Os itens são medidos numa escala de 5 pontos (desde discordo totalmente ( 0 pontos) a concordo totalmente (4 pontos) (Given et al., 1992; Nijboer, Triemstra, Tempelaar, Sandermanc, \& Bos, 1999). Um resultado elevado significa elevada sobrecarga. Os itens $2,3,15,21$ e 22 são cotados de forma invertida. Grov et al. (2006) ao realizarem a validação para a população de cuidadores informais de pacientes oncológicos noruegueses adicionaram uma escala total que correspondia à soma das subescalas do instrumento, estabelecendo correlações com outros instrumentos, sem prejuízo do conceito teórico subjacente. Deste modo, pode ser calculado um valor total, somandose os valores dos itens das subescalas.

Pereira e Sousa (2005) calcularam a fidelidade do instrumento numa amostra de 71 doentes com esclerose múltipla. O alfa obtido por dimensão foi: Auto estima (.67), Falta de Suporte Familiar (.83), impacto nas finanças (.80), impacto nos horários (.76) e impacto na saúde (.72). Devido ao tamanho da amostra não foi possível avaliar a validade do instrumento.

Para avaliar o Suporte Social utilizou-se a versão portuguesa da Instrumental and Expressive Social Support Scale (IESSS) (Faria, 1999) que tem o objectivo de quantificar a influência de acontecimentos de vida nos problemas de funcionamento expressivo e instrumental. O instrumento apresenta quatro subescalas: Vínculo Afectivo, Insatisfação com as Relações Íntimas, Conflito e Falta de Dinheiro, para além de permitir a utilização de um valor total que reflecte o suporte social em várias áreas, incluindo problemas financeiros, solicitações em função de tempo e esforço, existência de companhia adequada, comunicação, dependência, família e em problemas familiares quotidianos. O apoio social é compreendido como o acesso e 
utilização de recursos sociais para a preservação do próprio bem-estar do indivíduo. Por conseguinte, este instrumento reflecte a avaliação do apoio social percepcionado pela pessoa, permitindo compreender melhor a sua situação de vida. Este instrumento encontra-se validado na população portuguesa (Faria, 1999).

\section{Procedimento}

O processo de tradução e adaptação necessário para a utilização do Caregiver Reaction Assessment teve como base a orientação proposta por Bradley (1996) e, após consentimento da necessária autorização do autor de acordo com o seguinte procedimento:

a) Tradução pelos investigadores e simultaneamente por tradutor independente

b) Confronto das versões para elaboração da versão portuguesa

c) Retroversão por tradutor independente

d) Pré-teste com reflexão falada em 10 cuidadores informais para avaliar a adequação e compreensão dos itens

e) Elaboração da versão final do estudo.

A amostra foi recrutada junto de comunidades terapêuticas de tratamento de problemas de Dependência de Substâncias, de grupos de auto-ajuda a familiares de Dependentes de Substâncias e de serviços comunitários (escolas, universidades, grupos recreativos, etc.). Uma preocupação transversal a todo o projecto de investigação foi acautelar a compreensão dos procedimentos e dos objectivos por parte dos participantes e garantir a confidencialidade dos dados obtidos. Os questionários foram aplicados aos cuidadores, depois de fornecido, explicado e assinado o consentimento informado para a participação no estudo.

Foram definidos como critérios de exclusão: participantes que apresentavam algum problema oftalmológico não corrigido (por óculos ou lentes), apresentar história, sinais ou sintomas que possam ser suspeita da existência de Perturbação Esquizofrénica.

\section{RESULTADOS}

\section{Propriedade Psicométricas do Caregiver Reaction Assessment}

Versão Original

De acordo com os autores originais, esta escala apresenta uma boa fidelidade nas cinco dimensões: Auto-estima (0,90), Falta de suporte familiar (0,85), Finanças (0,81), Horários $(0,82)$ e Impacto na saúde $(0,80)$. A validade do construto foi conseguida com correlações modestas entre o CRA e medidas de depressão e dependência (Given et al., 1992; Kinsella, Cooper, Picton, \& Murtagh, 1998; Nijboer, et al., 1999). Numa amostra de cuidadores portugueses de pacientes com Esclerose Múltipla, a fidelidade do instrumento foi calculada pela computação do alfa de Cronbach. O alfa obtido por dimensão foi: Auto Estima $(0,67)$, Falta de 
Suporte Familiar (0,83), Impacto nas Finanças $(0,80)$, Impacto nos Horários $(0,76)$ e Impacto na Saúde $(0,72)$. No entanto, verificou-se que se retirasse, na dimensão Auto Estima, o item 12 melhoraria significativamente o alfa de Cronbach desta subescala. O mesmo acontece na dimensão Falta de Suporte Familiar com o item 2 e na dimensão Impacto nos Horários com o item 4 que se excluídos melhorariam as características psicométricas do instrumento (Sousa, 2005). Contudo as autoras, devido ao tamanho da amostra, não puderam realizar a validade do instrumento.

Amostra de Cuidadores de Dependentes de Substâncias

\section{Estudo de Fidelidade}

O estudo da consistência interna em relação às cinco subescalas do CRA, na amostra de cuidadores de Dependentes de Substâncias, está patente nos quadros 1 a 5.

\section{Quadro 1}

Coeficientes de consistência interna do CRA - 24 itens $(N=120)$ Sumário da subescala Auto Estima (7 itens) Média $=12.70 ;$ D.P. $=6.00 ;$ Alfa de Cronbach $=0.95$; Versão Original $=0.90$ )

\begin{tabular}{|l|c|c|c|c|}
\hline & $\begin{array}{c}\text { Média se } \\
\text { eliminado }\end{array}$ & $\begin{array}{c}\text { Variância se } \\
\text { eliminado }\end{array}$ & $\begin{array}{c}\text { Correlação com o } \\
\text { total (corrigido) }\end{array}$ & Alfa se eliminado \\
\hline CRA - item 1 & 11.23 & 25.63 & 0.87 & 0.93 \\
CRA - item 7 & 11.29 & 23.22 & 0.89 & 0.94 \\
CRA - item 9 & 10.33 & 24.69 & 0.87 & 0.94 \\
CRA - item 12 & 10.43 & 26.62 & 0.85 & 0.94 \\
CRA - item 17 & 10.50 & 29.65 & 0.73 & 0.95 \\
CRA - item 20 & 10.62 & 29.18 & 0.89 & 0.94 \\
CRA - item 23 & 11.80 & 27.86 & \\
\hline
\end{tabular}

A análise das correlações dos itens da subescala Auto Estima com o total da escala, revelou que todos os itens apresentam correlações acima dos 0,73, variando entre 0,73 e 0,90. Assim, verifica-se que a consistência interna não melhoraria com a eliminação de um qualquer destes itens, estando todos eles fortemente correlacionados com o total.

\section{Quadro 2}

Coeficientes de consistência interna do CRA - 24 itens $(N=120)$ Sumário da subescala Falta de Suporte Familiar (5 itens) Média = 9.35; D.P.= 4.40; Alfa de Cronbach = 0.80; Versão Original $=0.85$ )

\begin{tabular}{|l|c|c|c|c|}
\hline & $\begin{array}{c}\text { Média se } \\
\text { eliminado }\end{array}$ & $\begin{array}{c}\text { Variância se } \\
\text { eliminado }\end{array}$ & $\begin{array}{c}\text { Correlação com o total } \\
\text { (corrigido) }\end{array}$ & Alfa se eliminado \\
\hline CRA - item 2 & 7.14 & 15.62 & 0.23 & 0.87 \\
CRA - item 6 & 7.50 & 14.77 & 0.51 & 0.79 \\
CRA - item 13 & 7.95 & 11.80 & 0.69 & 0.73
\end{tabular}




\begin{tabular}{|l|l|l|l|l|} 
CRA - item 16 & 7.56 & 10.90 & 0.89 & 0.66 \\
CRA - item 22 & 7.25 & 12.06 & 0.72 & 0.72 \\
\hline
\end{tabular}

A análise das correlações dos itens da subescala Falta de Suporte Familiar com o total da escala revelou que todos os itens apresentam correlações acima dos 0,23 , variando entre 0,23 e 0,89 .

Quadro 3

Coeficientes de consistência interna do CRA - 24 itens $(\mathrm{N}=120)$ Sumário da subescala Impacto nos Horários (5 itens) Média = 9.21; D.P. = 4.31; Alfa de Cronbach = 0.82; Versão Original $=0.82$ )

\begin{tabular}{|l|c|c|c|c|}
\hline & $\begin{array}{c}\text { Média se } \\
\text { eliminado }\end{array}$ & $\begin{array}{c}\text { Variância se } \\
\text { eliminado }\end{array}$ & $\begin{array}{c}\text { Correlação com } \\
\text { o total } \\
\text { (corrioida) }\end{array}$ & $\begin{array}{c}\text { Alfa se } \\
\text { eliminado }\end{array}$ \\
\hline CRA - item 4 & 7.17 & 13.64 & 0.48 & 0.82 \\
CRA - item 8 & 7.50 & 11.43 & 0.71 & 0.75 \\
CRA - item 11 & 7.73 & 13.36 & 0.60 & 0.79 \\
CRA - item 14 & 7.69 & 14.06 & 0.48 & 0.81 \\
CRA - item 18 & 6.74 & 9.61 & 0.80 & 0.72 \\
\hline
\end{tabular}

A análise das correlações dos itens da subescala Impacto nos Horários com o total da escala, revelou que todos os itens apresentam correlações acima dos 0,80 , variando entre $0,80 \mathrm{e}$ 0,89 .

\section{Quadro 4}

Coeficientes de consistência interna do CRA - 24 itens ( $=120)$ Sumário da subescala Impacto na Saúde do Cuidador (4 itens) Média = 8.66; D.P. = 3.06; Alfa de Cronbach = 0.64 ; Versão Original $=0.80$ )

\begin{tabular}{|l|c|c|c|c|}
\hline & $\begin{array}{c}\text { Média se } \\
\text { eliminado }\end{array}$ & $\begin{array}{c}\text { Variância se } \\
\text { eliminado }\end{array}$ & $\begin{array}{c}\text { Correlação com o } \\
\text { total (corrigido) }\end{array}$ & $\begin{array}{c}\text { Alfa se } \\
\text { eliminado }\end{array}$ \\
\hline CRA - item 5 & 6.59 & 6.18 & 0.30 & 0.67 \\
CRA - item 10 & 6.53 & 5.93 & 0.32 & 0.66 \\
CRA - item 15 & 6.43 & 5.76 & 0.57 & 0.48 \\
CRA - item 19 & 6.42 & 5.72 & 0.57 & 0.48 \\
\hline
\end{tabular}

A análise das correlações dos itens da subescala Impacto na Saúde do Cuidador com o total da escala, revelou que todos os itens apresentam correlações acima dos 0,30 , variando entre 0,30 e 0,57 . 


\section{Quadro 5}

Coeficientes de consistência interna do CRA - 24 itens $(\mathrm{N}=120)$ Sumário da subescala Impacto nas Finanças (3 itens) Média = 7.18; D.P.= 2.40; Alfa de Cronbach = 0.57; Versão Original $=0.81$ )

\begin{tabular}{|l|c|c|c|c|}
\hline & $\begin{array}{c}\text { Média se } \\
\text { eliminado }\end{array}$ & $\begin{array}{c}\text { Variância se } \\
\text { eliminado }\end{array}$ & $\begin{array}{c}\text { Correlação com o } \\
\text { total (corrigido) }\end{array}$ & $\begin{array}{c}\text { Alfa se } \\
\text { eliminado }\end{array}$ \\
\hline CRA - item 3 & 4.26 & 2.75 & 0.64 & 0.09 \\
CRA - item 21 & 5.06 & 3.52 & 0.24 & 0.67 \\
CRA - item 24 & 5.03 & 3.09 & 0.32 & 0.58 \\
\hline
\end{tabular}

A análise das correlações dos itens da subescala Impacto nas Finanças com o total da escala, revelou que todos os itens apresentam correlações acima dos 0,24 , variando entre $0,24 \mathrm{e}$ 0,64 .

Como é visível, o estudo da consistência interna revelou que as subescalas Impacto na Saúde do Cuidador e Impacto nas Finanças apresentaram valores baixos (0,64 e 0,57). De seguida passou-se ao estudo de validade.

\section{Estudo de Validade}

O valor do teste de Kaiser-Meyer-Olkin (KMO) foi de 0,75, sendo considerável aceitável para a análise factorial. O teste de esfericidade de Bartlett foi significativo ao nível de 0,00 . Os resultados da análise factorial são apresentados no quadro 6 .

Para se realizar a validade da escala, primeiro procedeu-se à análise da medida de adequação da amostra (medida KMO), depois recorreu-se à análise factorial de cinco factores segundo a versão original. No entanto, os itens não apresentaram coerência com a versão original, nem com a versão adaptada à população portuguesa de cuidadores com esclerose múltipla. Posteriormente, realizou-se a análise factorial com 4 e 3 factores principais, sendo esta última a solução final adoptada, tendo em consideração a saturação dos itens e a coerência teórica e semântica. A opção de inclusão dos itens 3, 15, 19 nos respectivos componentes obedeceu a esse princípio. Assim, a rotação varimax com o pedido de supressão de cargas factoriais abaixo de 0,30 (usando o critério de Tinsley e Tinsley (1987) confirmou que os 3 factores principais explicavam $58,76 \%$ da variabilidade total.

Nesta versão adaptada à população portuguesa de cuidadores de Dependentes de Substâncias, o instrumento manteve os 24 itens, mas agora com três subescalas, sendo que todos os factores saturaram acima de 0,30 (cf. quadro 7). De acordo com Kline (1994) este valor de saturação das cargas é aceitável, uma vez que explicaria pelo menos $9 \%$ da variância total, sendo que Garson (2010) defende que a saturação deve ser utilizada em função da relevância teórica. 
A subescala anteriormente chamada de Auto Estima não sofreu alterações com a nova análise factorial, daí que optou-se por não alterar o nome da subescala, mantendo-se a sua dimensão positiva. Um baixo valor nesta subescala sugere um baixo valor atribuído ao acto de cuidar.

Os itens $4,5,6,8,11,13,15,16,18,22$ e 24 podem ser agrupados num factor chamado Impacto na Vida Pessoal e Familiar do Cuidador. Esta subescala pode ser definida como a percepção da responsabilidade do cuidador e dos seus familiares nos cuidados ao adicto e a influência da interferência nas actividades diárias (incluindo as relações familiares e o bem-estar subjectivo) dos cuidados prestados. De facto os itens abordam aspectos como: "As minhas actividades são centradas em redor no cuidar do meu companheiro(a)/familiar" (item 4) o que remete para a vivência do cuidador em termos pessoais e familiares. Um resultado baixo nesta subescala sugere um menor impacto no bem-estar do cuidador ao nível da vida pessoal e familiar.

Os itens 2, 3, 10, 14, 19 e 21 podem ser agrupados num factor chamado Impacto nas Finanças e Saúde do Cuidador. Pode ser definida essencialmente como a percepção que o cuidador tem da adequação dos recursos financeiros e da influência da tensão associada à prestação dos cuidados na sua saúde ("Os meus recursos financeiros são adequados para pagar o que é necessário para os cuidados ao meu familiar com DS - item 2). Um resultado baixo nesta subescala sugere um menor impacto nos recursos financeiros e saúde do cuidador como resultado de cuidar do seu familiar.

Ambas as subescalas correspondem à dimensão negativa da sobrecarga.

\section{Quadro 6}

Análise factorial do CRA - Matrix dos três componentes com rotação varimax e supressão de cargas factoriais abaixo de 0.30

\begin{tabular}{|l|c|c|c|}
\hline & \multicolumn{3}{|c|}{ Componentes } \\
\hline & 1- Auto-Estima & $\begin{array}{c}\text { 2-Impacto na Vida } \\
\text { Familiar do Cuidador }\end{array}$ & $\begin{array}{c}\text { 3 - Impacto na Saúde do } \\
\text { Cuidador }\end{array}$ \\
\hline CRA - item 1 & & 0.77 \\
CRA - item 2 & 0.85 & 0.57 & 0.35 \\
CRA - item 3 & & 0.43 & \\
CRA - item 4 & 0.38 & 0.45 & 0.33 \\
CRA - item 5 & 0.30 & 0.66 & 0.61 \\
CRA - item 6 & 0.88 & 0.55 & \\
CRA - item 7 & 0.36 & & \\
CRA - item 8 & 0.86 & 0.62 & \\
CRA - item 9 & 0.46 & & \\
CRA - item 10 & 0.41 & 0.67 & \\
CRA - item 11 & 0.88 & & \\
CRA - item 12 & & & \\
CRA - item 13 & & & \\
CRA - item 14 & & &
\end{tabular}




\begin{tabular}{|l|l|l|l|} 
CRA - item 15 & 0.49 & 0.48 & 0.53 \\
CRA - item 16 & & 0.69 & \\
CRA - item 17 & 0.77 & & 0.32 \\
CRA - item 18 & 0.34 & 0.66 & 0.43 \\
CRA - item 19 & 0.41 & 0.36 & 0.33 \\
CRA - item 20 & 0.66 & & \\
CRA - item 21 & & 0.81 & \\
CRA - item 22 & 0.90 & & \\
CRA - item 23 & & 0.58 & \\
CRA - item 24 & & & \\
\hline
\end{tabular}

Assim, nas tabelas 7 a 10 são apresentados, respectivamente, os coeficientes de consistência interna de Cronbach dos 24 itens do CRA, da subescala Auto Estima, Impacto na Vida Pessoal e Familiar do Cuidador e Impacto na Saúde do Cuidador.

\section{Quadro 7}

Coeficientes de consistência interna do CRA - 24 itens $(\mathrm{N}=120)$ Sumário da escala: Média $=47.09 ;$ D.P. $=16.15 ;$ Alfa de Cronbach $=0.95$

\begin{tabular}{|c|c|c|c|c|}
\hline & $\begin{array}{l}\text { Média se } \\
\text { eliminado }\end{array}$ & $\begin{array}{l}\text { Variância se } \\
\text { eliminado }\end{array}$ & $\begin{array}{l}\text { Correlação com o } \\
\text { total (corrigido) }\end{array}$ & $\begin{array}{c}\text { Alfa se } \\
\text { eliminado }\end{array}$ \\
\hline CRA - item 1 & 45.62 & 235.61 & 0.74 & 0.92 \\
\hline CRA - item 2 & 44.88 & 250.54 & 0.22 & 0.93 \\
\hline CRA - item 3 & 44.18 & 242.90 & 0.56 & 0.92 \\
\hline CRA - item 4 & 45.05 & 243.29 & 0.49 & 0.92 \\
\hline CRA - item 5 & 45.02 & 244.26 & 0.40 & 0.92 \\
\hline CRA - item 6 & 45.24 & 244.99 & 0.50 & 0.92 \\
\hline CRA - item 7 & 45.68 & 230.67 & 0.72 & 0.92 \\
\hline CRA - item 8 & 45.38 & 234.74 & 0.67 & 0.92 \\
\hline CRA - item 9 & 44.72 & 235.28 & 0.68 & 0.92 \\
\hline CRA - item 10 & 44.97 & 239.07 & 0.53 & 0.92 \\
\hline CRA - item 11 & 45.62 & 241.39 & 0.61 & 0.92 \\
\hline CRA - item 12 & 44.82 & 241.15 & 0.62 & 0.92 \\
\hline CRA - item 13 & 45.69 & 237.59 & 0.55 & 0.92 \\
\hline CRA - item 14 & 45.58 & 243.96 & 0.52 & 0.92 \\
\hline CRA - item 15 & 44.87 & 241.15 & 0.62 & 0.92 \\
\hline CRA - item 16 & 45.30 & 236.01 & 0.63 & 0.92 \\
\hline CRA - item 17 & 44.89 & 244.70 & 0.73 & 0.92 \\
\hline CRA - item 18 & 44.62 & 227.93 & 0.72 & 0.92 \\
\hline
\end{tabular}




\begin{tabular}{|l|l|l|l|l|} 
CRA - item 19 & 44.85 & 242.22 & 0.58 & 0.92 \\
CRA - item 20 & 45.01 & 241.89 & 0.75 & 0.92 \\
CRA - item 21 & 44.98 & 247.28 & 0.35 & 0.93 \\
CRA - item 22 & 44.99 & 237.68 & 0.59 & 0.92 \\
CRA - item 23 & 46.19 & 243.48 & 0.66 & 0.92 \\
CRA - item 24 & 44.95 & 240.68 & 0.51 & 0.92 \\
\hline
\end{tabular}

A análise das correlações dos itens do instrumento CRA com o total da escala, revelou que todos os itens apresentam correlações acima dos 0,22, variando entre 0,22 e 0,75.

\section{Quadro 8}

Coeficientes de consistência interna da Subescala: Auto Estima (7 itens) Sumário da escala: $\mathrm{N}=120$. Média $=12.70 ;$ D.P. $=6.00 ;$ Alfa de Cronbach $=0.95$

\begin{tabular}{|l|c|c|c|c|}
\hline & $\begin{array}{c}\text { Média se } \\
\text { eliminado }\end{array}$ & $\begin{array}{c}\text { Variância se } \\
\text { eliminado }\end{array}$ & $\begin{array}{c}\text { Correlação com o } \\
\text { total (corrigido) }\end{array}$ & $\begin{array}{c}\text { Alfa se } \\
\text { eliminado }\end{array}$ \\
\hline CRA - item 1 & 11.23 & 25.62 & 0.87 & 0.93 \\
CRA - item 7 & 11.29 & 23.21 & 0.89 & 0.93 \\
CRA - item 9 & 10.33 & 24.69 & 0.86 & 0.93 \\
CRA - item 12 & 10.43 & 26.61 & 0.84 & 0.93 \\
CRA - item 17 & 10.50 & 29.64 & 0.79 & 0.94 \\
CRA - item 20 & 10.62 & 29.18 & 0.73 & 0.94 \\
CRA - item 23 & 11.80 & 27.85 & 0.88 & 0.93 \\
\hline
\end{tabular}

A análise das correlações dos itens da subescala Auto Estima com o total da escala, revelou que todos os itens apresentam correlações acima dos 0,73 , variando entre 0,73 e 0,89 .

Quadro 9. Coeficientes de consistência interna da Subescala: Impacto na Vida Familiar do Cuidador (11 itens) Sumário da escala: $\mathrm{N}=120$. Média $=21.27 ;$ D.P. $=8.64 ;$ Alfa de Cronbach $=0.88$

\begin{tabular}{|l|c|c|c|c|}
\hline & $\begin{array}{c}\text { Média se } \\
\text { eliminado }\end{array}$ & $\begin{array}{c}\text { Variância se } \\
\text { eliminado }\end{array}$ & $\begin{array}{c}\text { Correlação com o } \\
\text { total (corrigido) }\end{array}$ & $\begin{array}{c}\text { Alfa se } \\
\text { eliminado }\end{array}$ \\
\hline CRA - item 4 & 19.22 & 65.57 & 0.45 & 0.88 \\
CRA - item 5 & 19.20 & 65.33 & 0.40 & 0.88
\end{tabular}




\begin{tabular}{|l|l|l|l|l|} 
CRA - item 6 & 19.42 & 64.81 & 0.57 & 0.87 \\
CRA - item 8 & 19.56 & 61.07 & 0.64 & 0.87 \\
CRA - item 11 & 19.79 & 63.94 & 0.62 & 0.87 \\
CRA - item 13 & 19.87 & 59.84 & 0.66 & 0.87 \\
CRA - item 15 & 19.04 & 64.36 & 0.59 & 0.87 \\
CRA - item 16 & 19.47 & 60.47 & 0.67 & 0.86 \\
CRA - item 18 & 18.80 & 56.93 & 0.73 & 0.86 \\
CRA - item 22 & 19.17 & 60.05 & 0.70 & 0.86 \\
CRA - item 24 & 19.12 & 63.30 & 0.52 & 0.87 \\
\hline
\end{tabular}

A análise das correlações dos itens da subescala Impacto na Vida Pessoal e Familiar do Cuidador com o total da escala, revelou que todos os itens apresentam correlações acima dos 0,41 , variando entre 0,41 e 0,74 .

\section{Quadro 10}

Coeficientes de consistência interna da Subescala: Impacto no Quotidiano do Cuidador (6 itens) Sumário da escala: N = 120. Média = 13.13; D.P. = 4.14; Alfa de Cronbach $=0.70$

\begin{tabular}{|l|c|c|c|c|}
\hline & $\begin{array}{c}\text { Média se } \\
\text { eliminado }\end{array}$ & $\begin{array}{c}\text { Variância se } \\
\text { eliminado }\end{array}$ & $\begin{array}{c}\text { Correlação com o } \\
\text { total (corrigido) }\end{array}$ & $\begin{array}{c}\text { Alfa se } \\
\text { eliminado }\end{array}$ \\
\hline CRA - item 2 & 10.92 & 12.76 & 0.32 & 0.69 \\
CRA - item 3 & 10.21 & 12.80 & 0.48 & 0.64 \\
CRA - item 10 & 11.00 & 11.42 & 0.50 & 0.63 \\
CRA - item 14 & 11.61 & 12.61 & 0.51 & 0.63 \\
CRA - item 19 & 10.88 & 13.04 & 0.44 & 0.65 \\
CRA - item 21 & 11.01 & 13.13 & 0.34 & 0.68 \\
\hline
\end{tabular}

A análise das correlações dos itens da subescala Impacto nas Finanças e Saúde do Cuidador com o total da escala revelou que todos os itens apresentam correlações acima dos 0,32 , variando entre 0,32 e 0,52 .

\section{Validade de Construto}

Em termos de validade de construto verificamos que a Sobrecarga correlaciona-se significativamente, de forma inversa, com o valor global de Suporte Social, i.e., a sobrecarga diminui com o aumento do suporte social $(r=-0.40, p<.01)$.

\section{Validade Discriminante}

A nossa amostra apresenta 53\% de cuidadores sobrecarregados e $47 \%$ de cuidadores sem sobrecarga, tendo-se utilizado o valor da mediana como ponto de corte (CRAtotal $=45$ ). 
No que diz respeito ao suporte social, os resultados indicam 9,7\% cuidadores com menor percepção de suporte social e 90,3\% de cuidadores com um nível de suporte social superior tendo em conta à média obtida na população portuguesa.

De seguida, avaliou-se a relação entre a sobrecarga e o suporte social nos sujeitos da amostra. Foram definidos dois grupos em função dos resultados obtidos em na escala de Suporte Social: $25 \%$ dos cuidadores com o valor mais elevado no IESSS (quartil superior) e $25 \%$ dos cuidadores com o valor mais baixo (quartil inferior).

$\mathrm{O}$ teste T de Student mostrou que os cuidadores de Dependentes de Substâncias com menor Suporte Social apresentam os valores mais elevados de Sobrecarga. As diferenças entre os dois grupos são estatisticamente significativas $(\mathrm{p}=0,00)$ (Quadro 11).

\section{Quadro 11}

Resultados do Teste T de Student, relativos à Sobrecarga, nos grupos de Cuidadores com Maior e Menor Suporte Social

\begin{tabular}{|l|c|c|c|c|c|c|}
\hline & \multicolumn{2}{|c|}{ Menor Suporte Social $(n=31)$} & Maior Suporte Social $(n=30)$ & \multicolumn{2}{c|}{ Teste $t$} \\
\hline & $M$ & $D . P$. & $M$ & $D . P$. & $t$ & $p$ \\
\hline Sobrecarga & 58.52 & 17.49 & 35.80 & 10.07 & 6.19 & 0.00 \\
\hline
\end{tabular}

\section{DISCUSSÃO}

Neste estudo pretendeu-se realizar a adaptação de uma medida de sobrecarga, o Caregiver Reaction Assessment (Given et al., 1992), numa amostra composta por cuidadores de Dependentes de Substâncias sendo que, na sua versão original, o autor realizou a análise psicométrica numa população de cuidadores de pacientes com Demência. No decurso deste processo deu-se relevo aos princípios estabelecidos por diversos autores, dos quais se salientam Bradley (1996), Hambleton e Patsula (1999) e Hambleton (2005), de forma a reduzir-se a possibilidade de erro. Deste modo, na tradução e adaptação as línguas e cultura de origem (inglês) e alvo (português) eram conhecidas, assim como o constructo e os princípios das boas práticas de desenvolvimento do teste. A decisão de se adaptar este instrumento foi tomada dado que existe a possibilidade de se realizarem estudos transculturais, para além do facto de que apresenta bons indicadores psicométricos na origem e no estudo piloto com cuidadores portugueses.

A adaptação da escala original, na presente amostra, revela uma escala com uma estrutura diferente que inclui em vez de cinco, três subescalas mantendo contudo os mesmos itens originais (24). A escala total adaptada apresenta elevada fidelidade, sendo que os três factores explicam 58,76\% da variabilidade total. A subescala Auto-Estima apresenta um $\alpha=0,95$, a subescala Impacto na Vida Familiar do Cuidador um $\alpha=0,88$ e a subescala Impacto no Quotidiano do Cuidador um $\alpha=0,70$. Os 24 itens do instrumento apresentam um $\alpha=0,95$, 
confirmando as boas qualidades psicométricas para ser utilizada numa população cuidadora de Dependentes de Substâncias, sendo útil como medida de sobrecarga.

Ao nível da validade de constructo verificou-se que a escala, como seria desejável, descrimina aos cuidadores com baixo versus alto suporte social e a sobrecarga correlaciona-se negativamente com a sobrecarga. Hanks et al. (2007) verificaram que o suporte social percebido está altamente relacionado com a percepção de sobrecarga, sendo o factor mais relevante na percepção da mestria enquanto cuidador e da satisfação da relação cuidador-paciente. Também, Majerovitz (2007) conseguiu determinar que o suporte social era um importante preditor da depressão em cuidadores, sendo crucial na atenuação da sobrecarga e na promoção da saúde mental. Gideon (2007) complementa esta noção ao ter verificado que as interacções sociais entre os cuidadores e a família e com os membros de outras redes sociais têm diferentes efeitos sobre o bem-estar emocional dos cuidadores. Assim, níveis mais elevados de suporte emocional por parte da família do cuidador tendem a reduzir o distress emocional do cuidador enquanto o suporte emocional por parte de membros das redes sociais informais tendem a melhorar a visão optimista da experiência de cuidar no cuidador.

Os resultados obtidos com a adaptação do CRA indicam tratar-se de um instrumento adequado para avaliar a sobrecarga em cuidadores de dependentes de substâncias. Ao nível da fidelidade, a escala apresenta bons s embora a subescala Impacto nas Finanças e Saúde do Cuidador seja a que apresenta uma inferior mas ainda assim aceitável (Nunally, 1987, Cronback, 1984). Contudo, segundo o autor referido, em contextos de aplicação, esta subescala é considerada limitada. Neste sentido, em situações de intervenção psicológica, em que os resultados ao nível da sobrecarga são usados para desenhar intervenções, o ideal será utilizar o resultado da escala global.

A análise factorial permitiu a identificação de 3 factores principais. A versão final inclui todos os 24 itens mantendo a integridade do instrumento original (anexo). Para além disso, o construto demonstrou validade ao correlacionar-se negativamente com o Suporte Social e discriminou com sucesso os cuidadores com maior sobrecarga.

No que diz respeito a investigações futuras, o estudo da eficácia de estratégias de gestão de stress, de estratégias de intervenção familiar e da relação entre as diferentes variáveis psicossociais que influenciam a sobrecarga nos cuidadores de Dependentes de Substâncias merecem uma atenção aprofundada. A literatura tem demonstrado que apesar de a população cuidadora de diferentes patologias apresentar semelhanças entre si, existem algumas diferenças nas consequências psicológicas, biológicas, familiares e sociais. Daí que, ao nível dos cuidadores de Dependentes de Substâncias, poderá ser útil discriminar a influência das características dos pacientes, do cuidador, do sistema familiar e das condições sociais ao nível do impacto na sobrecarga.

Apesar de neste trabalho se ter dado uma maior relevância á relação entre a sobrecarga e o suporte social na validade discriminativa, as intervenções orientadas para a atenuação dos 
efeitos da sobrecarga, devem privilegiar o papel de factores que também têm sido demonstrado como estando relacionados com a sobrecarga tal como o coping, o optimismo e a resiliência. De igual modo, a intervenção psicológica em cuidadores de dependentes de substâncias deve ser realizada por equipas multidisciplinares, entendendo o cuidador como um todo. O presente trabalho pretendeu apenas contribuir com um instrumento, em português, cuja adaptação reunisse as condições necessárias para a sua aplicação em cuidadores de dependentes de substancias na avaliação da sobrecarga, de modo a potenciar intervenções mais eficazes no seu alívio.

\section{REFERÊNCIAS}

Andrén, S., \& Elmståhl, S. (2007). Relationships between income, subjective health and caregiver burden in caregivers of people with dementia in group living care: A crosssectional community-based study. International Journal of Nursing Studies, 44(3), 435446.

Andrén, S., \& Elmståhl, S. (2008). The relationship between caregiver burden, caregivers' perceived health and their sense of coherence in caring for elders with dementia. Journal of Clinical Nursing, 17(6), 790-799. doi: 10.1111/j.1365-2702.2007.02066.x

Andrieu, S., Bocquet, H., Cantet, C., Grand, A., Balardy, L., Gillette-Guyonnet, S., et al. (2003). Informal caregiver's burden of Alzheimer's patients: Assessment and factors associated. Revue de Medecine Interne, 24(SUPPL. 3).

Awad, A. G., \& Voruganti, L. N. P. (2008). The burden of schizophrenia on caregivers: A review. PharmacoEconomics, 26(2), 149-162.

Bandeira, M., \& Barroso, S. M. (2005). Sobrecarga das famílias de pacientes psiquiátricos. Jornal Brasileiro de Psiquiatria, 54 (1), 34-46.

Beckham, J. C., Lytle, B. L., \& Feldman, M. E. (1996). Caregiver burden in partners of Vietnam War veterans with posttraumatic stress disorder. Journal of Consulting \& Clinical Psychology, 64(5), 1068-1072.

Bédard, M., Kuzik, R., Chambers, L., Molloy, D. W., Dubois, S., \& Lever, J. A. (2005). Understanding burden differences between men and women caregivers: The contribution of care-recipient problem behaviors. International Psychogeriatrics, 17(1), 99-118.

Belasco, A. G., \& Sesso, R. (2002). Burden and quality of life of caregivers for hemodialysis patients. American Journal of Kidney Diseases, 39(4), 805-812.

Black, W., \& Almeida, O. P. (2004). A systematic review of the association between the Behavioral and Psychological Symptoms of Dementia and burden of care. International Psychogeriatrics, 16(3), 295-315. 
Bradley, C. (1996). Translaction of Questionnaires for Use in Different Languages and Cultures. In C. Bradley (Ed.), Handbook of psychology and diabetes. A Guide to psychological measurement in Diabetes Research and Practice (pp. 43-56). London: Harwood Academic Publishers.

Bruce, J. M., McQuiggan, M., Williams, V., Westervelt, H., \& Tremont, G. (2008). Burden among spousal and child caregivers of patients with mild cognitive impairment. Dementia and Geriatric Cognitive Disorders, 25(4), 385-390. doi: 10.1159/000122587

Carter, B., \& McGoldrick, M. (Eds.). (1980). The Family Life Cycle: A framework for family therapy ( $1^{\mathrm{a}}$ ed.). New York: Gardner.

Chappell, N. L., \& Colin Reid, R. (2002). Burden and well-being among caregivers: Examining the distinction. Gerontologist, 42(6), 772-780.

Croog, S. H., Burleson, J. A., Sudilovsky, A., \& Baume, R. M. (2006). Spouse caregivers of alzheimer patients: Problem responses to caregiver burden. Aging \& Mental Health, 10(2), 87-100.

Dekel, R., \& Solomon, Z. (2007). Secondary traumatization among wives of war veterans with PTSD. In C. R. Figley \& W. P. Nash (Eds.), Combat stress injury: Theory, research, and management (Vol. 34, pp. 137-157). New York, NY: Routledge/Taylor \& Francis Group.

Demi, A., Bakeman, R., Moneyham, L., \& Sowell, R. (1997). Effects of resources and stressors on burden and depression of family members who provide care to an HIV-infected woman. Journal of Family Psychology, 11(1), 35-48.

Edwards, N. E., \& Scheetz, P. S. (2002). Predictors of burden for caregivers of patients with Parkinson's disease. The Journal of Neuroscience Nursing: Journal of the American Association of Neuroscience Nurses, 34(4), 184-190.

Faria, M. C. (1999). Escala de apoio social instrumental e expressivo - IESSS. In A. P. Soares, S. Araújo \& S. Caíres (Eds.), Avaliação psicológica: Formas e Contextos (Vol. VI, pp. 125-135). Braga: APPORT.

Garand, L., Eazor, L. R., Dew, M. A., DeKosky, S. T., \& Reynolds, C. F. (2005). Caregiving burden and psychiatric morbidity in spouses of persons with mild cognitive impairment. International Journal of Geriatric Psychiatry, 20(6), 512-522.

Garson, G. D. (2010). Factor Analysis Retrieved 1/10/2010, 2010, from http://faculty.chass.ncsu.edu/garson/PA765/factor.htm\#loading

Gideon, C. A. (2007). Social environments of dementia caregivers: Relationships between social support, negative social interactions, and caregiver emotional distress. Dissertation Abstracts International: Section B: The Sciences and Engineering, 67(7B), 0419-4217. 
Given, C. W., Given, B., Stommel, M., Collins, C., King, S., \& Franklin, S. (1992). The Caregiver Reaction Assessment (CRA) caregivers to persons with chronic physical and mental impairments. Research in Nursing \& Health, 15, 271-283.

Goldstein, N. E., Concato, J., Fried, T. R., Kasl, S. V., Bradley, E. H., \& Johnson-Hurzeler, R. (2004). Factors associated with caregiver burden among caregivers of terminally ill patients with cancer. Journal of Palliative Care, 20(1), 38-43.

Gómez-Ramos, M. J., \& González-Valverde, F. M. (2004). Caregivers of dementia patients: Application of the Caregiver Burden Scale. Revista Espanola de Geriatria $y$ Gerontologia, 39(3), 154-159.

Green, S. E. (2007). "We're tired, not sad": Benefits and burdens of mothering a child with a disability. Social Science \& Medicine, 64(1), 150-163.

Grov, E. K., Fosså, S. D., Sorebo, O., \& Dahl, A. A. (2006). Primary caregivers of cancer patients in the palliative phase: A path analysis of variables influencing their burden. Social Science \& Medicine, 63(9), 2429-2439.

Grov, E. K., Fosså, S. D., Tonnessen, A., \& Dahl, A. A. (2006). The Caregiver Reaction Assessment: Psychometrics, and Temporal Stability in Primary Caregivers of Norwegian Cancer Patients in Late Palliative Phase. Psycho-Oncology, 15(6), 517-527.

Hambleton, R. K. (2005). Adapting educational and psychological tests for cross-cultural assessment. In R. K. Hambleton, P. F. Merenda \& S. D. Spielberger (Eds.), Issues, designs and technical guidelines for adapting tests into multiple languages and cultures. (pp. 3-38). New Jersey: Lawrence Erlbaum Associates.

Hambleton, R. K., \& Patsula, L. (1999). Increasing the validity of adapted tests: Myths to be avoided and guidelines for improving test adaptation practices. Journal of Applied Testing Technology, 1, 1-30.

Hanks, R. A., Rapport, L. J., \& Vangel, S. (2007). Caregiving appraisal after traumatic brain injury: The effects of functional status, coping style, social support and family functioning. NeuroRehabilitation, 22(1), 43-52.

Jacobi, C. E., Rupp, I., van den Bos, G. A. M., van den Berg, B., Boshuizen, H. C., \& Dinant, H. J. (2003). Dimension-specific burden of caregiving among partners of rheumatoid arthritis patients. Rheumatology, 42(10), 1226-1233.

Jungbauer, J., \& Angermeyer, M. C. (2002). Living with a schizophrenic patient: A comparative study of burden as it affects parents and spouses. Psychiatry, 65(2), 110-123.

Kalra, H., Kamath, P., Trivedi, J. K., \& Janca, A. (2008). Caregiver burden in anxiety disorders. Current Opinion in Psychiatry, 21(1), 70-73.

Khan, F., Pallant, J., \& Brand, C. (2007). Caregiver strain and factors associated with caregiver self-efficacy and quality of life in a community cohort with multiple sclerosis. Disability and Rehabilitation: An International, Multidisciplinary Journal, 29(16), 1241-1250. doi: 10.1080/01443610600964141 
Kinsella, G. J., Cooper, B., Picton, C., \& Murtagh, D. (1998). A review of the measurement of caregiver and family burden in palliative care. Journal of Palliative Care, 14(2), 37-45.

Kline, P. (Ed.). (1994). An easy guide to factor analysis. New York: Routledge.

Kochar, J., Fredman, L., Stone, K. L., \& Cauley, J. A. (2007). Sleep problems in elderly women caregivers depend on the level of depressive symptoms: Results of the caregiver-study of osteoporotic fractures. Journal of the American Geriatrics Society, 55(12), 20032009.

La Parra, D. (2001). Contribution of women and low-income households to the provision of informal home healthcare. Gaceta sanitaria / S.E.S.P.A.S, 15(6), 498-505.

Lauber, C., Eichenberger, A., Luginbühl, P., Keller, C., \& Rössler, W. (2003). Determinants of burden in caregivers of patients with exacerbating schizophrenia. European Psychiatry, $18(6), 285-289$.

Loukissa, A. D. (1995). Family burden in chronic mental illness: a review of research studies. Journal of Advanced Nursing, 21, 248-255.

Magliano, L., Fadden, G., Economou, M., Held, T., Xavier, M., Guarneri, M., et al. (2000). Family burden and coping strategies in schizophrenia: 1-year follow-up data from the BIOMED I study. Social Psychiatry \& Psychiatric Epidemiology, 35(3), 109-115.

Magliano, L., Fiorillo, A., De Rosa, C., Malangone, C., Maj, M., Aletti, A., et al. (2004). Burden, attitudes and social support in the families of patients with long-term physical diseases. Epidemiologia e Psichiatria Sociale, 13(4), 255-261.

Majerovitz, S. D. (2007). Predictors of burden and depression among nursing home family caregivers. Aging \& Mental Health, 11(3), 323-329. doi: 10.1080/13607860600963380

Marsh, N. V., Kersel, D. A., Havill, J. H., \& Sleigh, J. W. (2002). Caregiver burden during the year following severe traumatic brain injury. Journal of Clinical and Experimental Neuropsychology, 24(4), 434-447.

Martens, L., \& Addington, J. (2001). The psychological wellbeing of family members of individual with schizophrenia. Social Psychiatry \& Psychiatric Epidemiology, 36(3), 128-133.

Martins, T., Ribeiro, J. P., \& Garrett, C. (2003). Estudo de Validação do Questionário de Avaliação da Sobrecarga para Cuidadores Informais. Psicologia, Saúde e Doenças, 4(1), 131-148.

Martire, L. M., Schulz, R., Reynolds, C. F., Morse, J. Q., Butters, M. A., \& Hinrichsen, G. A. (2008). Impact of close family members on older adults' early response to depression treatment. Psychology and Aging, 23(2), 447-452. doi: : 10.1037/0882-7974.23.2.447

Marvardi, M., Mattioli, P., Spazzafumo, L., Mastriforti, R., Rinaldi, P., Polidori, M. C., et al. (2005). The Caregiver Burden Inventory in evaluating the burden of caregivers of elderly demented 
patients: results from a multicenter study. Aging clinical and experimental research, 17(1), 4653.

Maurin, J. T., \& Boyd, C. B. (1990). Burden of mental illness on the family: A critical review. Archives of Psychiatric Nursing, 4(2), 99-107.

McClendon, M. J., \& Smyth, K. A. (2002). A longitudinal analysis of caregiver mental burden and well-being. Journal of Mental Health and Aging, 8(1), 19-28.

McGilloway, S., Donnelly, M., \& Mays, N. (1997). The experience of caring for former longstay psychiatric patients. British Journal of Clinical Psychology, 36, 149-151.

Meiland, F. J. M., Danse, J. A. C., Wendte, J. F., Klazinga, N. S., \& Gunning-Schepers, L. J. (2001). Caring for relatives with dementia - Caregiver experiences of relatives of patients on the waiting list for admission to a psychogeriatric nursing home in the Netherlands. Scandinavian Journal of Public Health, 29(2), 113-121.

Nijboer, C., Triemstra, M., Tempelaar, R., Sandermanc, R., \& Bos, G. A. M. v. d. (1999). Measuring both negative and positive reactions to giving care to cancer patients: Psychometric qualities of the Caregiver Reaction Assessment (CRA). Social Science \& Medicine, 48, 1259-1269.

O'Rourke, N., \& Tuokko, H. A. (2004). Caregiver burden and depressive symptomatology: The association between constructs over time. Clinical Gerontologist, 27(4), 41-52.

Ohaeri, J. U. (2003). The burden of caregiving in families with a mental illness: A review of 2002. Current Opinion in Psychiatry, 16(4), 457-465.

Paúl, C. (1997). Lá para o fim da vida: Idosos, família, e meio ambiente. Coimbra: Almedina.

Pickett, T. J. (2007). Caregiver burden in Huntington's disease. Dissertation Abstracts International: Section B: The Sciences and Engineering, 67(8-B), 4720.

Pinquart, M., \& Sörensen, S. (2003). Associations of stressors and uplifts of caregiving with caregiver burden and depressive mood: A meta-analysis. Journals of Gerontology Series B Psychological Sciences and Social Sciences, 58(2).

Post, M. W. M., Bloemen, J., \& De Witte, L. P. (2005). Burden of support for partners of persons with spinal cord injuries. Spinal Cord, 43(5), 311-319.

Rammohan, A., Rao, K., \& Subbakrishna, D. K. (2002). Religious coping and psychological well-being in carers of relatives with schizophrenia. Acta Psychiatrica Scandinavica, 105, 356-362.

Reine, G., Duplan, S., Lançon, C., Avorio, A., Siméoni, M. C., Auquier, P., et al. (2004). Evaluation of burden in caregivers of patients suffering from schizophrenia. Dimensions and domains. Annales Medico-Psychologiques, 162(6), 453-461.

Riebschleger, J., Scheid, J., Luz, C., Mickus, M., Liszewski, C., \& Eaton, M. (2008). How are the experiences and needs of families of individuals with mental illness reflected in medical education guidelines? Academic Psychiatry Special Issue: Reaching out to families and overcoming stigma, 32(2), 119-126. doi: 10.1176/appi.ap.32.2.119 
Rose, L. E. (1996). Families of psychiatric patients: a critical review and future research directions. Archives of Psychiatric Nursing, 10(2), 67-76.

Schrag, A., Hovris, A., Morley, D., Quinn, N., \& Jahanshahi, M. (2006). Caregiver-burden in Parkinson's disease is closely associated with psychiatric symptoms, falls, and disability. Parkinsonism and Related Disorders, 12(1), 35-41.

Schulze, B., \& Rössler, W. (2005). Caregiver burden in mental illness: Review of measurement findings and interventions in 2004-2005. Current Opinion in Psychiatry, 18(6), 684691.

Sousa, C. (2005). Representações da doença, Ajustamento à doença, Morbilidade Psicológica, Qualidade de Vida e Satisfação Marital em doentes com Esclerose Múltipla e seus Cuidadores. Mestrado, Universidade do Minho, Braga.

St. Onge, M., \& Lavoie, F. (1997). The experience of caregiving among mothers o adults suffering from psychoric disorders: factor associated to their psychological distress. American Journal of Community Psychology, 25(1), 73-94.

Stancin, T., Wade, S. L., Walz, N. C., Yeates, K. O., \& Taylor, H. G. (2008). Traumatic brain injuries in early childhood: Initial impact on the family. Journal of Developmental \& Behavioral Pediatrics, 29(4), 253-261. doi: 10.1097/DBP.0b013e31816b6b0f

Tessler, R. C., \& Gamache, G. M. (Eds.). (2000). Family Experiences with Mental lllness. Westport: Auburn House.

Tinsley, H. E. A., \& Tinsley, D. J. (1987). Uses of factor analysis in counseling psychology research. Journal of Counseling Psychology, 34(4), 414-424.

Wolff, N., Perlick, D. A., Kaczynski, R., Calabrese, J., Nierenberg, A., \& Miklowitz, D. J. (2006). Modeling Costs and Burden of Informal Caregiving for Persons with Bipolar Disorder. Journal of Mental Health Policy and Economics, 9(2), 99-110.

Yeh, S. H., Johnson, M. A., \& Wang, S. T. (2002). The changes in caregiver burden following nursing home placement. International Journal of Nursing Studies, 39(6), 591-600.

\section{ANEXO 1}

\section{CAREGIVER REACTION ASSESSMENT INSTRUMENT (CRA)}

(Given et al., 1992)

Instruções: Pretendemos conhecer como cuidar de um familiar com dependência de substâncias (DS) pode afectar o cuidador, a sua família e as rotinas diárias. Por favor seleccione a resposta que melhor representa como se sente em relação a cada afirmação tendo em conta o período da semana passada.

\begin{tabular}{|l|l|l|l|l|l|}
\hline & $\begin{array}{c}\text { Discordo } \\
\text { Totalmente }\end{array}$ & Discordo & $\begin{array}{c}\text { Nem Discordo nem } \\
\text { Concordo }\end{array}$ & $\begin{array}{c}\text { Concordo } \\
\text { Totalmente }\end{array}$ \\
\hline $\begin{array}{l}\text { 1. Sinto-me privilegiado(a) por cuidar do } \\
\text { meu familiar com DS. }\end{array}$ & & & & & \\
\hline $\begin{array}{l}\text { 2. Os outros atiraram para cima de mim a } \\
\text { responsabilidade de cuidar do meu } \\
\text { familiar com DS. }\end{array}$ & & & & & \\
\hline $\begin{array}{l}\text { 3. Os meus recursos financeiros são } \\
\text { adequados para pagar o que é necessário } \\
\text { para os cuidados ao meu familiar com }\end{array}$ & & & & & \\
\hline
\end{tabular}




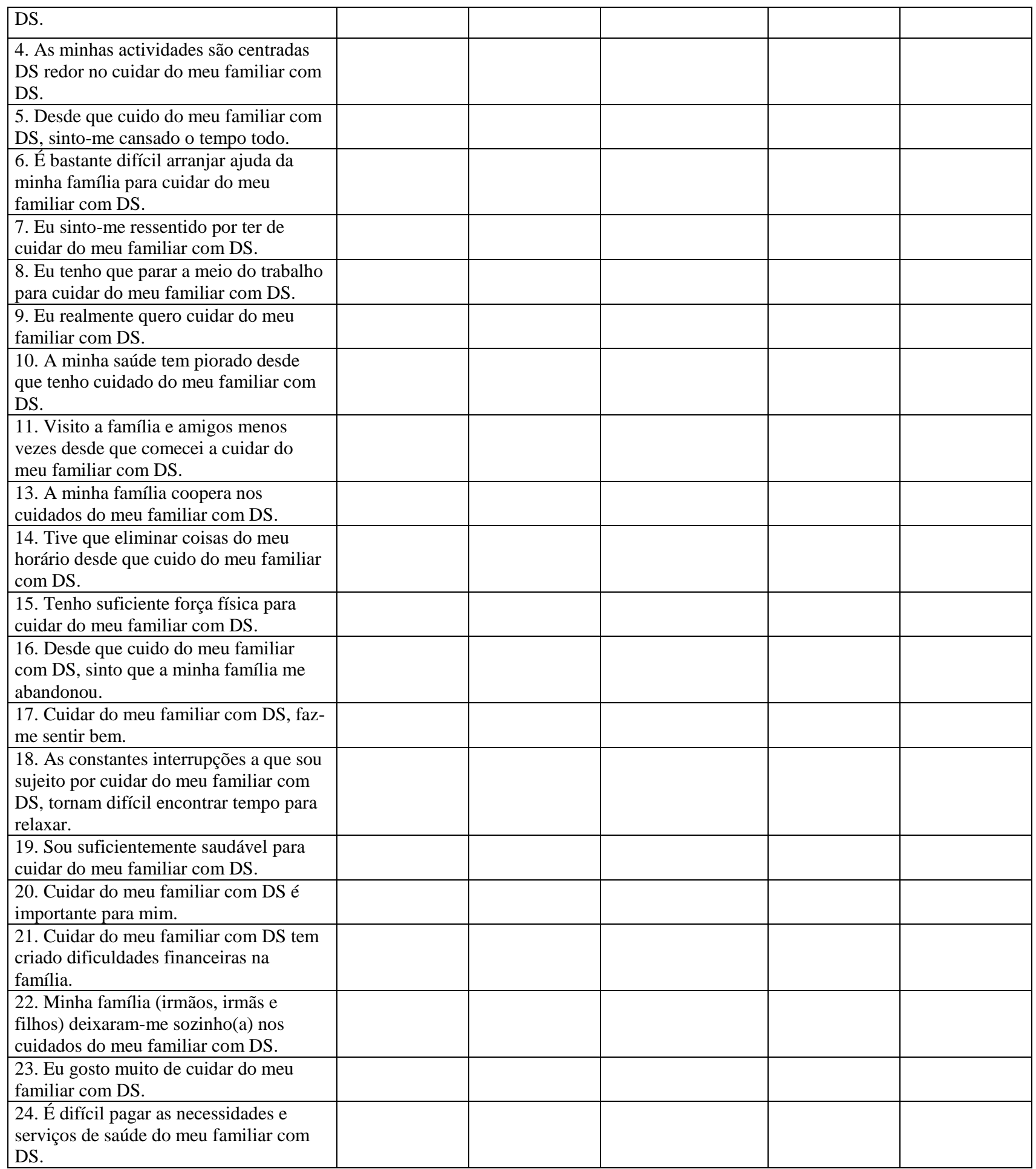

